\title{
Polymeric-lens-embedded 2D/3D switchable display with dramatically reduced crosstalk
}

\author{
Ruidong Zhu, ${ }^{1}$ Su Xu, ${ }^{1}$ Qi Hong, ${ }^{1}$ Shin-Tson Wu, ${ }^{1, \star}$ Chiayu Lee, ${ }^{2}$ Chih-Ming Yang, ${ }^{2}$ \\ Chang-Cheng Lo, ${ }^{2}$ and Alan Lien ${ }^{3}$ \\ ${ }^{1}$ CREOL, The College of Optics and Photonics, University of Central Florida, Orlando, Florida 32816, USA \\ ${ }^{2}$ China Star Optoelectronics Technology, Shenzhen, Guangdong, China \\ ${ }^{3} \mathrm{TCL}$ Corporate Research, Guangdong, China \\ *Corresponding author: swu@ucf.edu
}

Received 29 November 2013; revised 18 January 2014; accepted 23 January 2014; posted 27 January 2014 (Doc. ID 202113); published 26 February 2014

\begin{abstract}
A two-dimensional/three-dimensional (2D/3D) display system is presented based on a twisted-nematic cell integrated polymeric microlens array. This device structure has the advantages of fast response time and low operation voltage. The crosstalk of the system is analyzed in detail and two approaches are proposed to reduce the crosstalk: a double lens system and the prism approach. Illuminance distribution analysis proves these two approaches can dramatically reduce crosstalk, thus improving image quality. (C) 2014 Optical Society of America

OCIS codes: (110.1080) Active or adaptive optics; (110.0110) Imaging systems; (100.6890) Threedimensional image processing.

http://dx.doi.org/10.1364/AO.53.001388
\end{abstract}

\section{Introduction}

Two-dimensional/three-dimensional (2D/3D) switchable auto-stereoscopic displays have useful applications in home theaters and mobile displays. A critical element in the 2D/3D switchable display is the adaptive liquid crystal (LC) lens array [ $\underline{1}-\underline{5}]$. To achieve a short focal length for a given lens aperture, the cell gap of the LC layer is usually quite thick, which causes sluggish response time and increased operation voltage. For example, for an LC lens with a $30 \mu \mathrm{m}$ cell gap, the response time is about $1 \mathrm{~s}$ [6]. To reduce response time, several methods have been proposed, such as using a high birefringence LC to reduce cell gap [7], a Fresnel lens instead of a conventional LC lens [ $[-10]$, a polymer network LC [11], and by applying overdrive and undershoot voltages $[\underline{12}, \underline{13}]$.

$1559-128 \mathrm{X} / 14 / 071388-08 \$ 15.00 / 0$

(C) 2014 Optical Society of America
In addition to response time, two additional bottlenecks for the lens-based 2D/3D switchable display system are crosstalk and viewing angle [14-16], which will greatly degrade the image quality. Many approaches have been proposed to reduce crosstalk. Some of them are based on image processing [17], while the mainstream approach is based on optimizing the optical system, such as a specifically designed pixel mask for projection display [18], combined lens structures $[19,20]$, pixel optimization by modifying the layout of light blocking components [21], and using triplet structures for reducing crosstalk at large angles [22].

In this paper, we focus on solving the response time and crosstalk issues of the 2D/3D display system. To improve response time, we demonstrate a polymeric lenticular microlens array integrated with a twistednematic (TN) polarization rotator to indirectly actuate the lens. Such a microlens array can be switched from a nonfocusing to a focusing state (focal length $\sim 4 \mathrm{~mm}$ ) in $\sim 10 \mathrm{~ms}$, which is at least ten times 
faster than that of a conventional lens. We implemented this approach to design a 55 in. $(139.7 \mathrm{~cm})$ ultrahigh definition (UHD: $3840 \times 2160$ pixels) $2 \mathrm{D} /$ $3 \mathrm{D}$ switchable display and simulated its crosstalk. A detailed analysis of crosstalk is presented. To reduce crosstalk, we propose two methods: a double lens system and the prism approach. Based on the ray tracing results, our approaches can dramatically reduce the crosstalk.

\section{Lens Structure}

Figure 1 depicts the operation principles of our TNcell-based polymeric lens array. The TN cell functions as a polarization rotator. In the null voltage state [Fig. 1(a)], the incident linearly polarized light is not focused because its polarization is rotated by $90^{\circ}$ through the $\mathrm{TN}$ cell and becomes perpendicular (o-ray) to the optical axis of the polymeric lens. In a high voltage state $(\sim 5 \mathrm{~V})$, the polarization rotation effect vanishes as the LC directors are reoriented along the electric field direction. The outgoing beam from the TN cell acts as an e-ray for the polymeric lens. As a result, it is focused, as Fig. 1(b) shows. Because the switching between $2 \mathrm{D}$ and $3 \mathrm{D}$ displays is governed by the thin $\mathrm{TN}$ cell, the response time and driving voltage are superior to those of a conventional LC lens.

In our experiment, we prepared a polymeric microlens array and a TN cell separately. To fabricate the polymeric lens, we followed the procedures described by Ren et al. [4]. We used fringing fields generated from a planar top indium tin oxide (ITO) electrode and interdigitated bottom ITO electrodes to create the desired gradient refractive index profile for each microlens. The bottom striped electrodes have $50 \mu \mathrm{m}$ width and $410 \mu \mathrm{m}$ gap. The cell gap was controlled at $71 \mu \mathrm{m}$ by spacers. The LC/monomer mixture we employed consisted of 20 wt. \% BL003 $(\Delta \mathrm{n}=0.261$, $\mathrm{n}_{0}=1.531$, and $\left.\Delta \varepsilon=17\right)$ and 80 wt. \% RM257 $\left(\Delta \mathrm{n}=0.179, \mathrm{n}_{0}=1.508\right.$, and $\left.\Delta \varepsilon=-1.5\right)$. This mixture shows several desirable features at room temperature: (1) positive $\Delta \varepsilon(\sim 2.2),(2) \Delta \mathrm{n} \sim 0.195$, and (3) a better flexibility after UV curing. The mixture was filled into a homogeneous cell at $\sim 75^{\circ} \mathrm{C}$. After UV curing, the polymeric lens was cooled down to room temperature, peeled off from the substrates, and laminated to the TN cell.

The TN cell was filled with Merck E7 LC mixture $(\Delta \mathrm{n}=0.225$, viscosity $=39 \mathrm{cP}$, and $\Delta \varepsilon=13.8)$, and
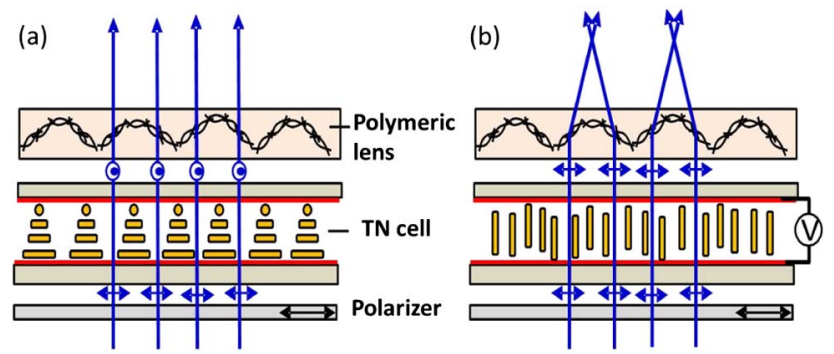

Fig. 1. (a) Nonfocusing state and (b) focusing state of the proposed LC polymeric lens incorporated with a TN cell.
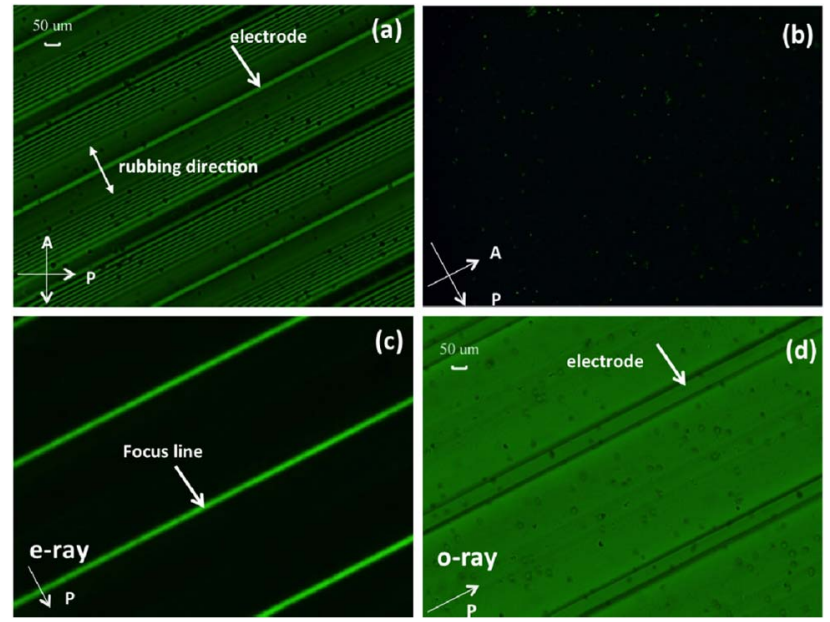

Fig. 2. (a) Interference patterns observed under a polarized optical microscope (POM) after UV curing. The film axis is at $45^{\circ}$ to the optical axis of the polarizer. (b) Dark state observed under a POM. The film axis is at $0^{\circ}$ to the optical axis of the polarizer. (c) Parallel focused lines. The film axis is parallel to the optical axis of the polarizer (no analyzer). (d) Uniform bright state. The film axis is perpendicular to the optical axis of the polarizer (no analyzer).

the cell gap was $5 \mu \mathrm{m}$. With $5 \mathrm{~V}$ of driving voltage, the response time from nonfocusing to focusing states was measured to be $3.7 \mathrm{~ms}$, while from focusing to nonfocusing states was $13 \mathrm{~ms}$. Such a response time is at least ten times faster than that of a conventional LC lens [6]. If we use the thin cell approach as reported in [7], the response time can be further reduced to $\sim 1 \mathrm{~ms}$.

Figure 2 shows some experimental results of the polymeric lens that we fabricated. Figure 2(a) shows the interference pattern observed under a polarized optical microscope (POM) after UV curing at $55 \mathrm{~V}_{\text {rms }}$. The rubbing direction was set at $45^{\circ}$ with respect to the optical axis of the polarizer. We can see 11.5 pairs of black and white fringes, which imply a $4 \mathrm{~mm}$ focal length based on following lens equation:

$$
f=\pi r^{2} / \varphi \lambda,
$$

where $r$ is the aperture radius $(230 \mu \mathrm{m}), \varphi$ is the phase difference between the lens border and lens center, and $\lambda$ is the wavelength of the illumination light $(546 \mathrm{~nm})$. The measured focal length is $\sim 3.73 \mathrm{~mm}$ and we can fine-tune it to $4 \mathrm{~mm}$ by reducing the applied voltage during UV curing. The cell was then rotated by another $45^{\circ}$ so that its rubbing direction was parallel to the optical axis of the polarizer. A very dark state is obtained [Fig. 2(b)], and here the light leakage comes from the spacers. Removing the analyzer and adjusting the film position, parallel focused lines were clearly observed [Fig. 2(c)]. When the cell is rotated by $90^{\circ}$, i.e., its rubbing direction is perpendicular to the optical axis of polarizer, a bright uniform texture is observed [Fig. 2(d)]. As explained before, the polymeric film presents a uniform refractive index to the o-ray. These experimental results 
demonstrate that our lens structure can be switched between focusing and nonfocusing states.

\section{Interpretation of the LC Lens}

The performance of the polymer lens array can greatly affect the quality of a 2D/3D display. However, there is no direct way to verify every aspect of the imaging performance of the polymer lens at this moment. In order to integrate it into a ray tracing system for performance evaluation, a translation from the polymer lens to an equivalent solid lens is required, which is based on the equal phase retardation assumption. In this way, we can evaluate how the defects in the polymer lens affect the display performance. For the polymer lens, the phase retardation $(\varphi)$ across the lens aperture is determined by the cell gap $(d)$ and effective refraction index distribution $\left(n_{\text {eff }}\right)$ as [23]

$$
\varphi=2 \pi n_{\mathrm{eff}} d / \lambda .
$$

While for a solid lens, the phase retardation has following form:

$$
\varphi=2 \pi\left[n d_{0}-(n-1) \Delta d\right] / \lambda,
$$

where $n$ is the refractive index of the solid material, $d_{0}$ is the lens thickness at the center, and $\Delta d$ is the lens thickness distribution across the lens aperture. Comparing Eqs. (2) and (3), we can translate the LC lens into an equivalent solid lens using following two equations:

$$
\begin{gathered}
n_{0} d=n d_{0}, \\
\Delta n d=(n-1) \Delta d,
\end{gathered}
$$

where $n_{0}$ is the effective refractive index at the center of the polymer lens and $\Delta n$ is the effective refractive index distribution of the polymer lens across the whole aperture.

Let us take Fig. 3(a) as an example, which shows the DIMOS-calculated and parabolic-fitted phase profile of our polymeric lens. It is obvious that at the edge of the LC cell, the phase profile has a small "tail" and slightly deviates from the parabolic shape. However, if we translate the lens phase profile into a solid lens shown in Fig. 3(b) and use a ray tracing software to evaluate its performance, we can see that the deviation does not have much impact on the 3D display, which will be explained later.

To quantitatively evaluate how the lens profile deviates from the ideal parabolic shape, the error function (EF) of the lens profile is introduced [24], which describes the difference between the actual phase profile $\left(S_{i}\right)$ and the ideal parabolic phase profile $\left(P_{i}\right)$. Its mathematical definition is

$$
E F=\sqrt{\sum\left(S_{i}-P_{i}\right)^{2} / D} \times 100 \%,
$$
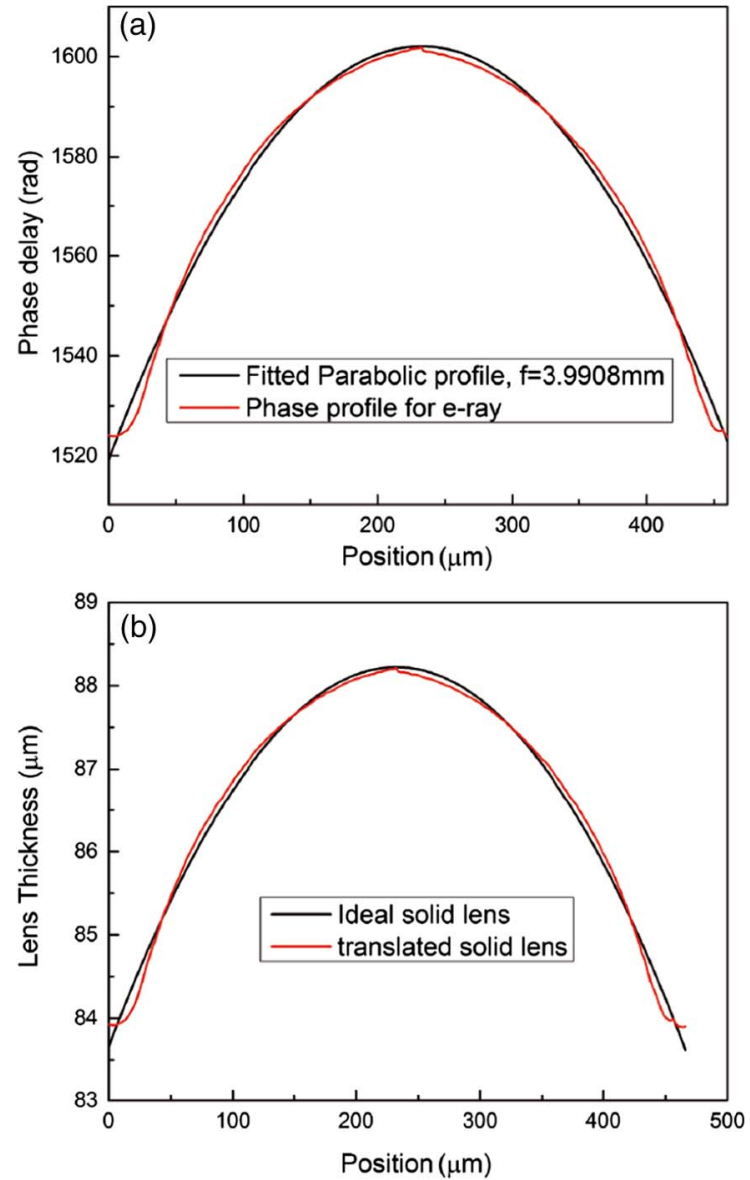

Fig. 3. (a) Simulated phase profile of the polymer lens by DIMOS and the fitted parabolic profile, and (b) the translated solid lens profile.

where $D$ is the aperture size and the summation is calculated across the whole lens aperture. A larger $\mathrm{EF}$ indicates that the lens has larger aberration and higher crosstalk. Our lenticular lens has an $\mathrm{EF}$ of $4.52 \%$, which means its lens profile doesn't deviate much from the parabolic shape. The EF function can also be extrapolated for other types of phase profile, such as elliptical or hyperbolic.

\section{2D/3D Switchable Display System and its Crosstalk Analysis}

Although the focal length of our polymeric lens shown in Fig. 2 was $\sim 3.73 \mathrm{~mm}$, it can be increased to the desired $\sim 4 \mathrm{~mm}$ by slightly reducing the applied voltage, which is applicable for large panel 2D/3D switchable displays. To prove this concept, we propose a $55 \mathrm{in}$. UHD 2D/3D switchable display. The system configuration is shown in Table 1 [25]. Based on this configuration, we build a 9 -view $\overline{2 \mathrm{D}}$ / $3 \mathrm{D}$ switchable display.

When evaluating an autostereoscopic display, the most important parameter is crosstalk, which is defined as the light illuminance penetrated from the adjacent view into the contemporary viewing zone at the sweet spot (best viewing position) [21], namely 
Table 1. Parameters of the Autostereoscopic Display

\begin{tabular}{lcc}
\hline Setup & Specification & Characteristic \\
\hline Display panel & resolution & $3840 \times 2160$ \\
& size & (UHD) \\
& pixel dimension & $315 \mu \mathrm{m} \times 315 \mu \mathrm{m}$ \\
& pixel density & $80 \mathrm{PPI}$ \\
Lenticular lens & lens pitch & $466 \mu \mathrm{m}$ \\
& focal length $(f)$ & $3.99 \mathrm{~mm}$ \\
& lens thickness & $80 \mu \mathrm{m}$ \\
Autostereoscopic & slanted angle & $9.46^{\circ}$ \\
display & viewing distance & $2.48 \mathrm{~m}$ \\
& number of views & 9 \\
& interval of viewpoints & $32.5 \mathrm{~mm}$ \\
\hline
\end{tabular}

$$
\mathrm{X}=I_{\min } /\left(I_{\min }+I_{\max }\right) \times 100 \% .
$$

The definition of crosstalk is depicted in Fig. 4(a). The alignment between the polymer lens array and the pixel plane is shown in Fig. 4(b). The slanted lines indicate the edges of the polymer lens and the numbers indicate which view the subpixel belongs to. From Fig. 4(b), we can deduce that a large portion of light from View 4 and View 6, together with a small portion of light from View 3 and View 7 , will leak to View 5 . This is proven by the normalized illuminance distribution of the system shown in Fig. $4(\mathrm{c})$. For View 5, at the sweet spot $x=0 \mathrm{~mm}$, a large portion of the light from View 4 and View 6 penetrates into the spot, while the light from View 3 and View 7 is negligible. From Fig. 4(c), we can deduce that each viewing zone is $62 \mathrm{~mm}$ (FWHM) wide and the average view-to-view crosstalk of the system is $34 \%$.

Such a high crosstalk would cause severe image degradation in 3D displays, thus it has to be reduced. Crosstalk may come from several aspects, such as misalignment between the lens array and the pixel plane, thermal expansion of the lens array after long-term use [19], as well as lens profile deformation introduced during fabrication process [26]. To simplify the discussion, let us assume the lens is well fabricated and the alignment is perfect so that we will focus on optimizing the display panel and lens array to reduce crosstalk. Figure $\underline{5}$ lists these factors that may affect the crosstalk of our system.

The layout of an actual subpixel is shown in Fig. 6(a). The aperture ratio is $\mathrm{AR}=48.7 \%$, which is much smaller than our previous assumption $(\mathrm{AR}=100 \%)$. The effective pixel size is greatly reduced and thus a narrower view with smaller overlap between adjacent views is achieved. This time, each viewing zone width is $50 \mathrm{~mm}$ and the average viewto-view crosstalk is $16.7 \%$, as shown in Fig. 6(b).

Although reducing the AR of a display panel can greatly reduce the crosstalk, it lowers the transmittance. Moreover, the light blocking parts will also be imaged onto the viewing plane, thus the light intensity distribution will not be uniform. One can even

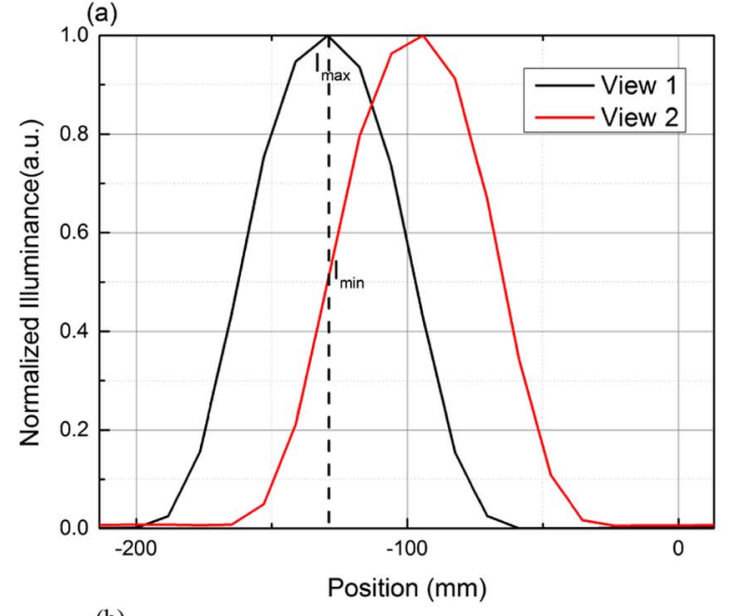

(b)

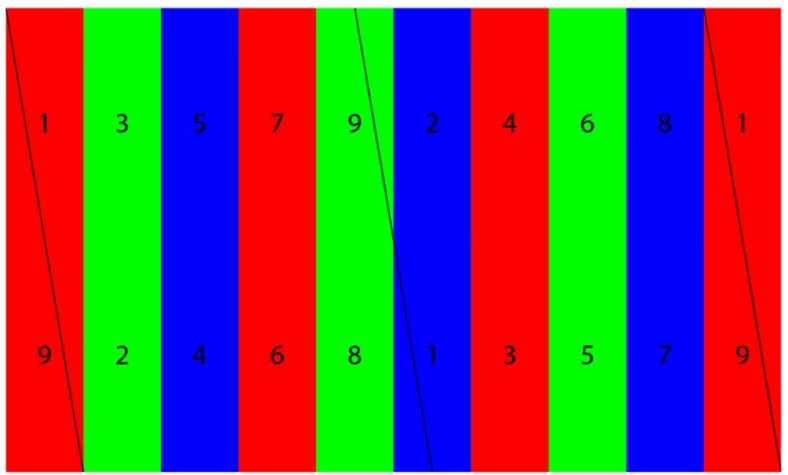

(c)

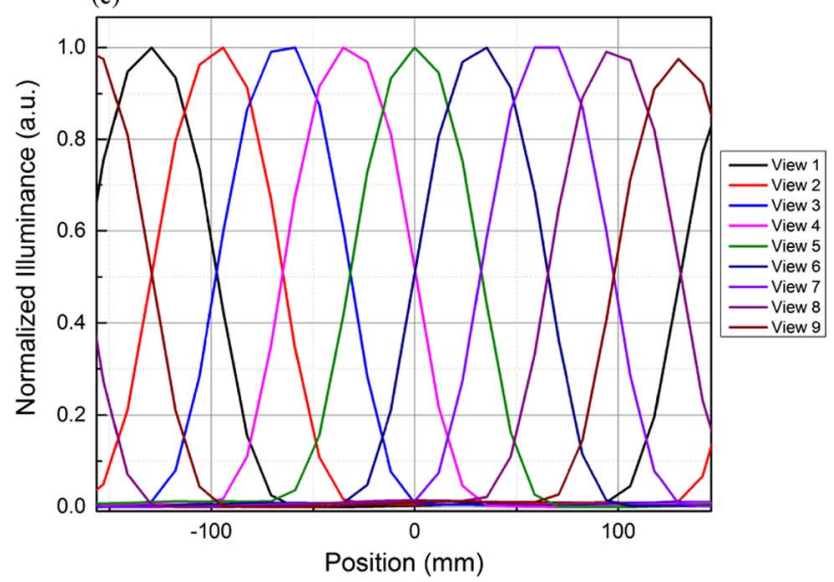

Fig. 4. (a) Definition of crosstalk, (b) the alignment between the pixel plane and the slanted lens, and (c) normalized illuminance distribution of the autostereoscopic display.

see dark bands when moving from one side to another side of the display. This trade-off of smaller $\mathrm{AR}$ can be evaluated by the intensity modulation depth [27]. Given this reason, further reducing AR is not a favorable solution for crosstalk reduction.

In addition, many researchers have focused on fine-tuning the lens profile to reduce the crosstalk, such as using aspherical lens for a better focusing [14]. Its design procedure is explained as follows: a distant point source produces collimated rays, the rays are then refracted by the lens, and the lens 


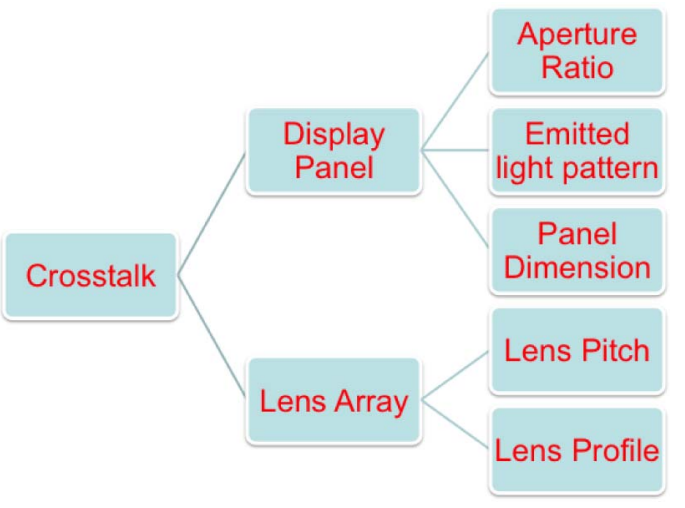

Fig. 5. Some factors that may lead to crosstalk.

(a)

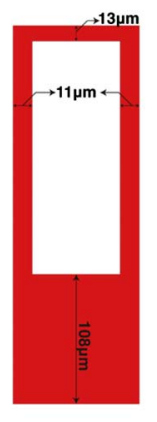

$$
\text { (b) }
$$

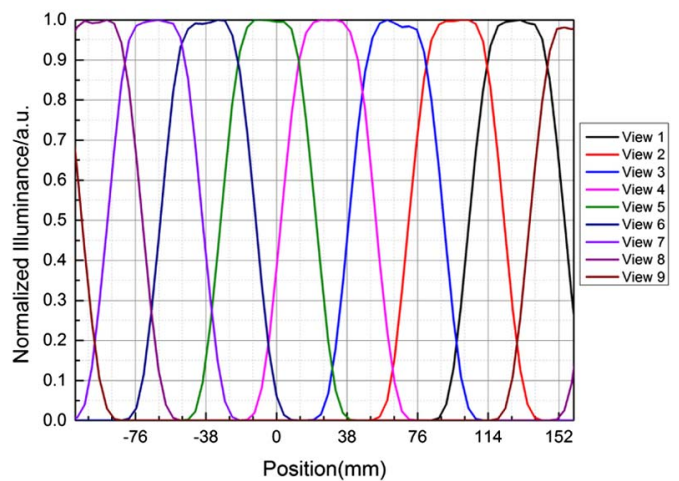

Fig. 6. (a) Proposed pixel dimension, and (b) normalized illuminance distribution $(\mathrm{AR}=48.7 \%)$.

profile is optimized to get to the smallest focus spot on the back focal plane. Then, because of the reversibility of the optical system, pixels on the back focal plane will be imaged on the viewing plane.

Figure 7 shows an example of the procedure. The two lenticular lenses shown in Figs. 7(a) and 7(b) both have a $4 \mathrm{~mm}$ focal length, but their cross sections are different [Fig. 7(c)]. It is obvious that the lens shown in Fig. 7(b) has a better focusing ability than that in Fig. 7(a), especially at large off-axis angles. However, when the two lens arrays are individually integrated with the display system, their light distribution of a single view are quite similar, only the peak positions are shifted, as Fig. 7(d) shows. One can see that fine-tuning lens profile does not improve the illuminance distribution noticeably. The possible reasons are: (1) This approach usually requires a lens pitch to be tuned together with the lens profile, which is usually not the case for our display as the width of the lens pitch is quite rigid to ensure each slanted lens covers 9 views. (2) This optimization method assumes that each subpixel can be regarded as a point source, which is indeed an extended source with a unique light intensity distribution pattern [21].

As each subpixel is regarded as extended light source, each subpixel is collimated by a few adjacent lenses, which results in several lobes. In fact, when

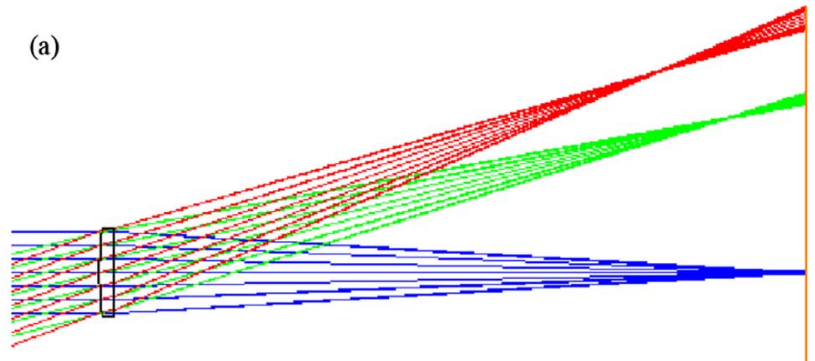

(b)
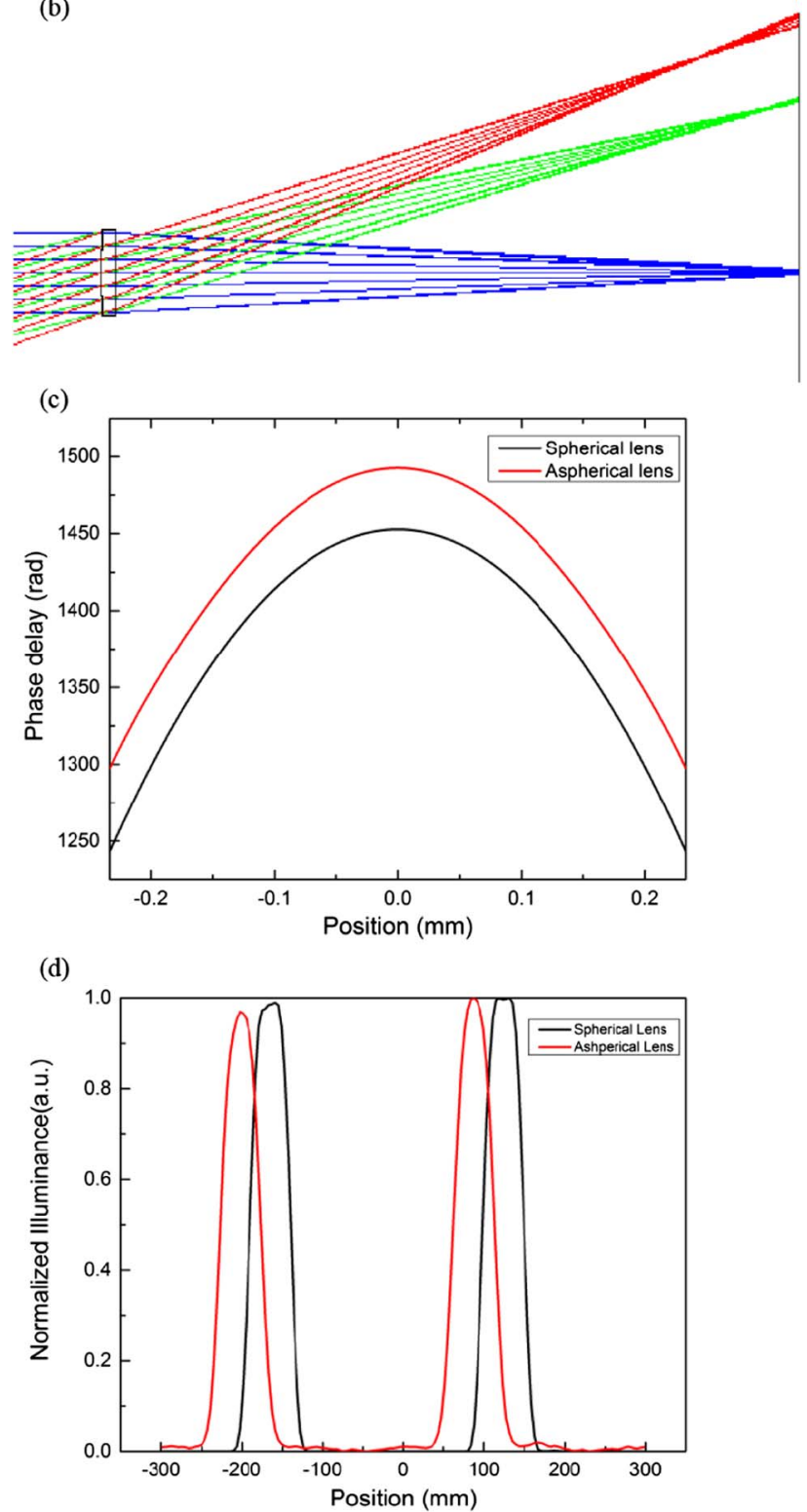

Fig. 7. Performance of (a) a spherical lens and (b) an aspherical lens. (c) The profile and (d) the normalized illuminance of the two lenses.

the focusing power of the LC lens is acceptable (the $\mathrm{EF}$ of the structure is small), the light distribution of each lobe at the optimized viewing plane is mainly 


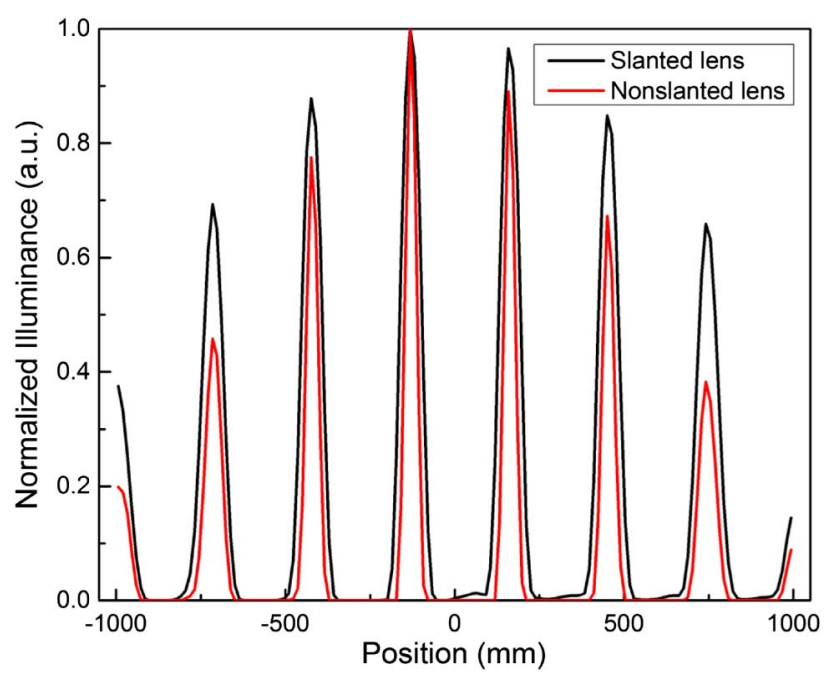

Fig. 8. Comparison between slanted and nonslanted system.

determined by the effective pixel dimension and the lens pitch of the slanted lens. The slanted lens array has the ability to average the light distribution among different lobes and thus makes each lobe smoother. This is demonstrated in Fig. 8, where the light illuminance of View 1 after the slanted lens array is shown in black while the light illuminance after an equivalent nonslanted lens array (same theoretical viewing zone width but the pitch is $0.945 \mathrm{~mm}$ ) is indicated by the red line. It is obvious that the viewing zone is narrower for the nonslanted lens, and at the same time the light illuminance is more concentrated in the main lobes. The narrower viewing zone usually comes with a smaller overlap between adjacent views and thus smaller crosstalk.

These reasons can also explain why the tail at the edge of our lens profile does not degrade the lens performance that much, because the error function is small (EF 4.52\%) and the slanted lens averages the light illuminance distribution.

\section{Double-Lens System for Crosstalk Reduction}

From the above discussion, it can be seen that it is difficult to further reduce the system crosstalk using a single lens array. The first reason is that light from the display panel is not collimated, while most lens optimization approaches are only applicable for collimated light, such as the achromatic lens array approach and the nonspherical lens array approach. The second reason is that the crosstalk mainly depends on the viewing zone width of each view, which is determined by the effective pixel dimension and the lens pitch, and has been predetermined here. Given these two reasons, we propose a double-lens array structure to further reduce the crosstalk, as shown in Fig. 9.

The first lens array (focal length $1 \mathrm{~mm}$ ) is placed at $3 \mathrm{~mm}$ in front of the display panel, and the second lens array (focal length $4 \mathrm{~mm}$ ) is located at $5.5 \mathrm{~mm}$ away from the first lens array. The viewing plane is located at $2480 \mathrm{~mm}$ away from the display

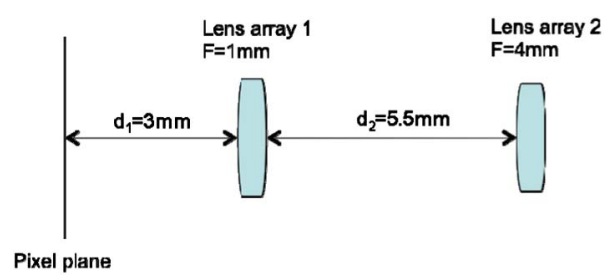

array

Pixel plane

tem.

panel. The first lens array works as a "shrinking" lens to reduce the effective pixel dimension, as the image of the pixel plane is located at $1.5 \mathrm{~mm}$ behind the first lens and $4 \mathrm{~mm}$ in front of the second lens array. Thus, the second lens array works as a focusing lens array to achieve a 3D effect. The normalized illuminance distribution is shown in Fig. 10. At the sweet spot, the light leakage from adjacent views is dramatically reduced. Using our proposed double lens system, the average view-to-view crosstalk is reduced to $3 \%$, and the average viewing zone width is $32 \mathrm{~mm}$. The trade-off is that at the sweet spot the normalized illumination distribution is not as flat as that shown in Fig. 6(b). This means that the system's moving freedom is limited, but at the sweet spot the viewing experience is greatly enhanced.

The optical path of the double-lens system is a little bit long for practical application. However, in a real system many other components are inserted between the display panel and the first lens array, such as glass substrates and polarizers, so the real path length can be reduced as all these components have relatively high refractive index. Moreover, the required thickness of the display modules can be reduced by using a smaller F\# lens array. If the focal length of the first lens array can be reduced to $0.5 \mathrm{~mm}$ (F\# 4.76), then the total optical path length can be reduced from 8.5 to $6.25 \mathrm{~mm}\left(\mathrm{~d}_{1}=1.5 \mathrm{~mm}\right.$ and $\mathrm{d}_{2}=4.75 \mathrm{~mm}$ ). We can fine-tune the double-lens system by the following design principle: the first lens array is employed to decrease the effective pixel dimension of the display panel, and the second lens array is utilized to achieve 3D effect.

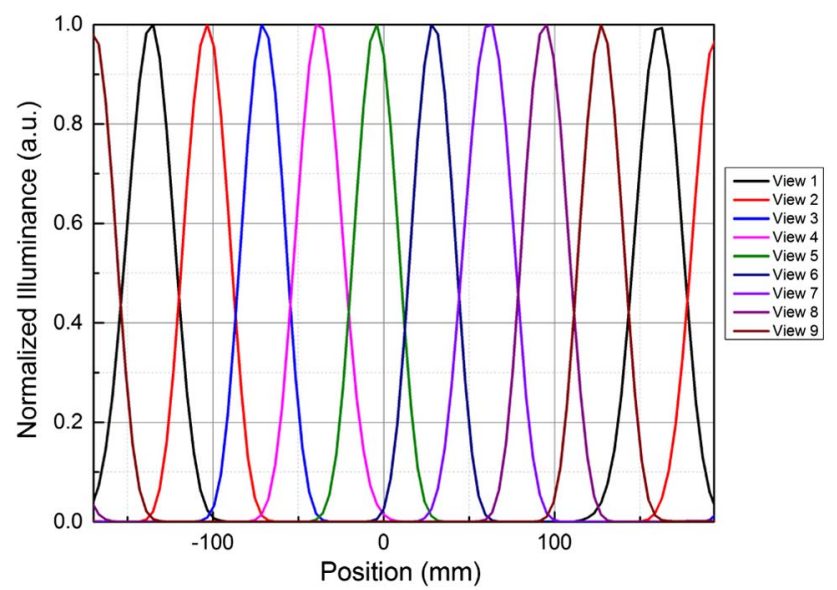

Fig. 10. Normalized illuminance distribution of the double lens structure. 


\section{Prism Approach}

For a large display panel, such as a 55 in. UHD display, the display panel size also affects the crosstalk. For example, Fig. 11 shows the normalized light illuminance distribution of View 5 . The black curve represents the normalized illuminance of the light emitted from the subpixel in the middle of the panel, and the red curve represents the normalized illuminance of the light emitted from the subpixel on the right side of the panel. We can see that each peak in these two curves shifts (about $16 \mathrm{~mm}$ ), and at $x=$ $-750 \mathrm{~mm}$ (the left side of the viewing plane), the light almost vanishes for the pixels on the right. This means that on the left side of the viewing plane, it is impractical to see images of the subpixel on the right side of the panel. The peak shift and illuminance drop will introduce larger viewing zone width and higher crosstalk, while light vanishes on the left side implies to a smaller viewing angle. Such a phenomenon also happens to the left subpixels and makes the problem even worse. To solve this problem, we propose the prism approach to shift the light ray to wherever we need on the viewing plane.

Figure 12(a) shows a specially designed prism for pixels on the right side of the panel. The pitch of the prism remains the same $(466 \mu \mathrm{m})$. The design principle is to shift the maximum illuminance peak of the light emitted from the subpixel on the right side of the panel (presented by the red curve in Fig. 11) from the right side to near the center of the display panel, and viewers on the left side can have a good view of pixels on the right side. The profiles of the two prisms are shown in Fig. 12(b). If we use an LC lens, we can meet this requirement by translating the prism profile into an equivalent LC phase profile. We design two LC prisms, and Fig. 12(c) illustrates the normalized illuminance of the light emitted from the subpixel on the right side of the panel after using the LC prism. It is obvious that the prism has the ability to deviate the peaks to different positions, and the

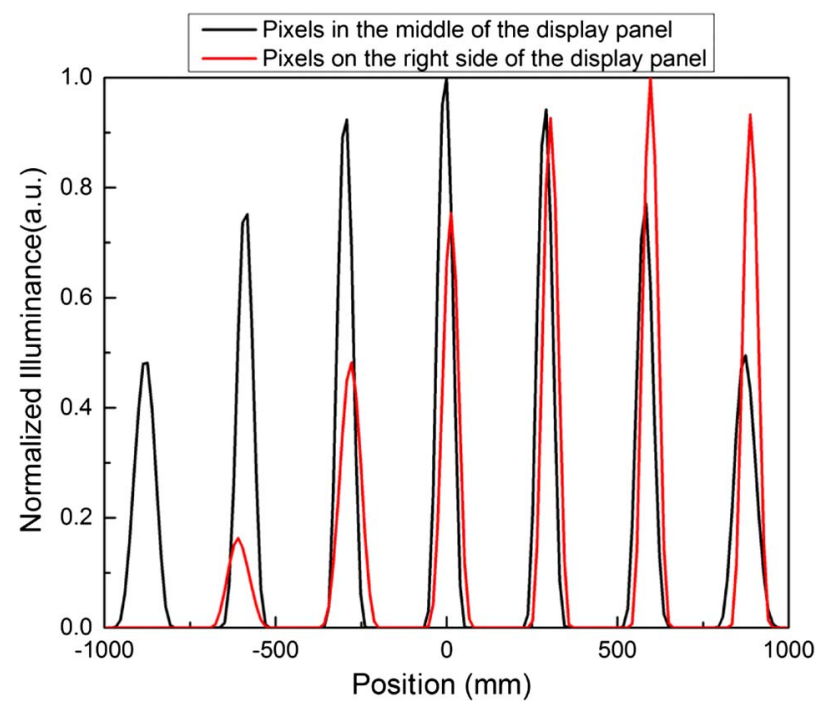

Fig. 11. Normalized illuminance distribution of View 5 . (a)

(b)
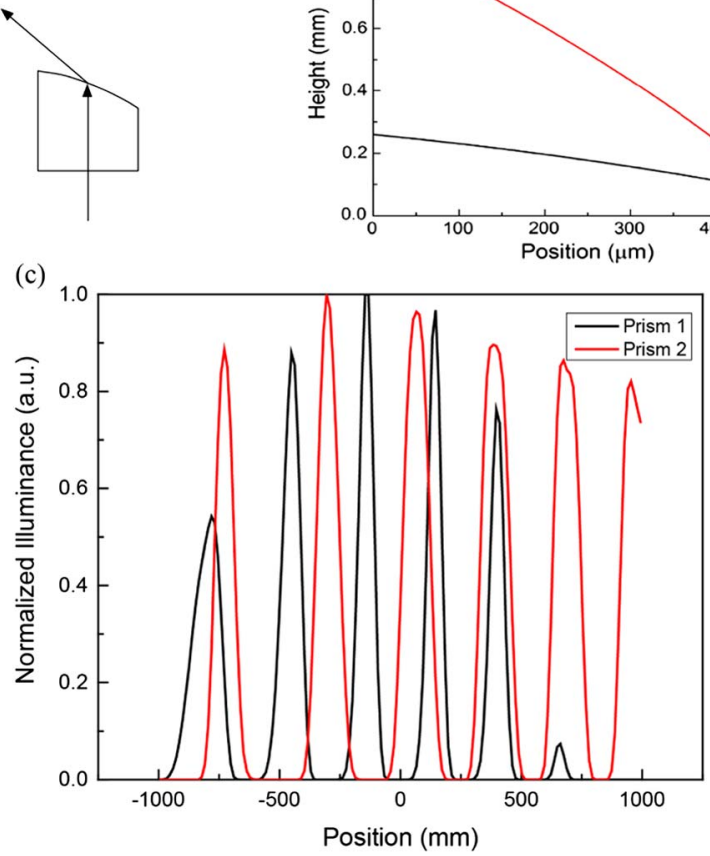

Fig. 12. (a) Schematic design of the LC prism, (b) prism profile for a large display panel, and (c) light illuminance redistribution by using different LC prisms.

illuminance on the left side of the viewing plane (e.g., $x=-750 \mathrm{~mm}$ ) is still acceptable for both Prisms 1 and 2, which means a larger viewing angle. For Prism 1, the on-axis performance is degraded because on the right side the peak is very small, while for Prism 2 a balance between the on-axis and offaxis light distribution can be achieved by optimizing its curvature. The illumination peak can be further tuned to the center of the display panel.

\section{Conclusion}

We propose a TN cell integrated polymeric lens, whose focal length can be switched from infinity to $4 \mathrm{~mm}$ and response time is about $10 \mathrm{~ms}$, which is at least ten times faster than a conventional LC lens. The polymeric lens array is then integrated into a 55 in. UHD display and the crosstalk of the system is analyzed in detail. Two approaches are presented to reduce the crosstalk of the system: the double lens structure and the prism approach.

The authors are indebted to Y. Liu and Z. Luo of UCF for useful discussion and China Star Optoelectronics Technology for financial support.

\section{References}

1. J. Hong, Y. Kim, H. J. Choi, J. Hahn, J. H. Park, H. Kim, S. W. Min, N. Chen, and B. Lee, "Three-dimensional display technologies of recent interest: principles, status, and issues [Invited],” Appl. Opt. 50, H87-H115 (2011).

2. G. J. Woodgate and J. Harrold, "Key design issues for autostereoscopic 2-D/3-D displays," J. Soc. Inf. Disp. 14, 421-426 (2006). 
3. C. van Berkel, "Image preparation for 3D LCD," Proc. SPIE 3639, 84-91 (1999).

4. H. Ren, S. Xu, Y. Liu, and S.-T. Wu, "Switchable focus using a polymeric lenticular microlens array and a polarization rotator," Opt. Express 21, 7916-7925 (2013).

5. H. Urey, K. V. Chellappan, E. Erden, and P. Surman, "State of the art in stereoscopic and autostereoscopic displays," Proc. IEEE 99, 540-555 (2011).

6. H. Ren and S.-T. Wu, "Adaptive liquid crystal lens with large focal length tunability," Opt. Express 14, 11292-11298 (2006).

7. S. Gauza, X. Zhu, W. Piecek, R. Dabrowski, and S.-T. Wu, "Fast switching liquid crystals for color-sequential LCDs," J. Display Technol. 3, 250-252 (2007).

8. G. Q. Li, D. L. Mathine, P. Valley, P. Ayras, J. N. Haddock, M. S. Giridhar, G. Williby, J. Schwiegerling, G. R. Meredith, B. Kippelen, S. Honkanen, and N. Peyghambarian, "Switchable electro-optic diffractive lens with high efficiency for ophthalmic applications," Proc. Natl. Acad. Sci. USA 103, 6100-6104 (2006).

9. H. Ren, Y.-H. Fan, and S.-T. Wu, "Tunable Fresnel lens using nanoscale polymer-dispersed liquid crystals," Appl. Phys. Lett. 83, 1515-1517 (2003).

10. Y.-P. Huang, C.-W. Chen, and Y.-C. Huang, "Superzone fresnel liquid crystal lens for temporal scanning auto-stereoscopic display," J. Display Technol. 8, 650-655 (2012).

11. J. Sun, S. Xu, H. Ren, and S.-T. Wu, "Reconfigurable fabrication of scattering-free polymer network liquid crystal prism/ grating/lens," Appl. Phys. Lett. 102, 161106 (2013).

12. S. T. Wu and C. S. Wu, "Small angle relaxation of highly deformed nematic liquid crystals," Appl. Phys. Lett. 53, 1794-1796 (1988).

13. Y. Liu, H. Ren, S. Xu, Y. Chen, L. Rao, T. Ishinabe, and S.-T. $\mathrm{Wu}$, "Adaptive focus integral image system design based on fast-response liquid crystal microlens," J. Display Technol. 7, 674-678 (2011).

14. R. B. Johnson and G. A. Jacobsen, "Advances in lenticular lens arrays for visual display," Proc. SPIE 5874, 587406 (2005).

15. A. J. Woods, "Crosstalk in stereoscopic displays: a review," J. Electron. Imaging 21, 040902 (2012).

16. K. H. Lee, Y. Park, H. Lee, S. K. Yoon, and S. K. Kim, "Crosstalk reduction in auto-stereoscopic projection 3D display system," Opt. Express 20, 19757-19768 (2012).
17. X. Li, Q. Wang, Y. Tao, D. Li, and A. Wang, "Crosstalk reduction in multi-view autostereoscopic three-dimensional display based on lenticular sheet," Chin. Opt. Lett. 9, 021001 (2011).

18. T.-H. Hsu, M.-H. Kuo, H.-H. Huang, S.-C. Chuang, C.-H. Chen, and C.-H. Tsai, "50.4: High resolution autostereoscopic 3D display with proximity projector array," SID Int. Symp. Dig. Tech. Pap. 39, 760-763 (2008).

19. W. X. Zhao, Q. H. Wang, A. H. Wang, and D. H. Li, "Autostereoscopic display based on two-layer lenticular lenses," Opt. Lett. 35, 4127-4129 (2010).

20. A. H. Wang, Q. H. Wang, X. F. Li, and D. H. Li, "Combined lenticular lens for autostereoscopic three dimensional display," Optik 123, 827-830 (2012).

21. C.-H. Chen, Y.-P. Huang, S.-C. Chuang, C.-L. Wu, H.-P. D. Shieh, W. Mphep, C.-T. Hsieh, and S.-C. Hsu, "Liquid crystal panel for high efficiency barrier type autostereoscopic threedimensional displays," Appl. Opt. 48, 3446-3454 (2009).

22. H. Kim, J. Hahn, and H. J. Choi, "Numerical investigation on the viewing angle of a lenticular three-dimensional display with a triplet lens array," Appl. Opt. 50, 1534-1540 (2011).

23. Y.-P. Huang, L.-Y. Liao, and C.-W. Chen, "2-D/3D switchable autostereoscopic display with multi-electrically driven liquid-crystal (MeD-LC) lenses," J. Soc. Inf. Disp. 18, 642646 (2010).

24. C.-W. Chen, Y.-C. Huang, Y.-P. Huang, and J.-F. Huang, "30.1: Fast switching Fresnel liquid crystal lens for autostereoscopic 2D/3D display," SID Int. Symp. Dig. Tech. Pap. 41, 428-431 (2010).

25. J. H. Jung, J. Yeom, J. Hong, K. Hong, S. W. Min, and B. Lee, "Effect of fundamental depth resolution and cardboard effect to perceived depth resolution on multi-view display," Opt. Express 19, 20468-20482 (2011).

26. M. Nirmal, C. A. Marttila, E. A. Aho, G. E. Casner, E. M. Haus, C. O. Schwabacher, and A. M. Schwabacher, "23.2: Design, fabrication, and characterization of multi-view, glasses free, 3D displays," SID Int. Symp. Dig. Tech. Pap. 43, 305-307 (2012).

27. M. Sluijter, A. Herzog, D. K. G. de Boer, M. P. C. M. Krijn, and H. P. Urbach, "Ray-tracing simulations of liquid-crystal gradient-index lenses for three-dimensional displays," J. Opt. Soc. Am. B 26, 2035-2043 (2009). 